\title{
A Sequential Monte Carlo Approach for Online Stock Market Prediction Using Hidden Markov Models
}

\author{
Ahani E. Bridget \\ University of Lagos, briggetk2002ng@yahoo.com \\ O. Abass \\ University of Lagos, Nigeria, Africa, olabass@unilag.edu.ng
}

Follow this and additional works at: http:// digitalcommons.wayne.edu/jmasm

Part of the Applied Statistics Commons, Social and Behavioral Sciences Commons, and the Statistical Theory Commons

\section{Recommended Citation}

Bridget, Ahani E. and Abass, O. (2011) "A Sequential Monte Carlo Approach for Online Stock Market Prediction Using Hidden Markov Models," Journal of Modern Applied Statistical Methods: Vol. 10 : Iss. 2 , Article 25.

DOI: $10.22237 /$ jmasm/1320121440

Available at: http://digitalcommons.wayne.edu/jmasm/vol10/iss2/25

This Regular Article is brought to you for free and open access by the Open Access Journals at DigitalCommons@WayneState. It has been accepted for inclusion in Journal of Modern Applied Statistical Methods by an authorized editor of DigitalCommons@WayneState. 


\title{
A Sequential Monte Carlo Approach for Online Stock Market Prediction Using Hidden Markov Models
}

\author{
Ahani E. Bridget O. Abass \\ University of Lagos, \\ Nigeria, Africa
}

A sequential Monte Carlo (SMC) algorithm prediction approach is developed based on joint probability distribution in hidden Markov Models (HMM). SMC methods, a general class of Monte Carlo methods, are typically used for sampling from sequences of distributions and simple examples of these algorithms are found extensively throughout the tracking and signal processing literature. Recent developments indicate that these techniques have much more general applicability and can be applied very effectively to statistical inference problems. Due to the problem involved in estimating the parameter of HMM, the HMM is represented in a state space model and the sequential Monte Carlo (SMC) method is used. Predictions are made using the SMC method in HMM and the corresponding on-line algorithm is developed. Daily stock price data from the banking sector of the Nigerian Stock Exchange (NSE) (price index between the years 1 January 2005 to 31 December 2008) are analyzed; experimental results reveal that the method proposed is effective.

Key words: Sequential Monte Carlo, hidden Markov model, state-space model, stock market.

\section{Introduction}

State space, or hidden Markov models (HMM), are convenient means to statistically model a process that varies over time. The state space model (Doucet\& Johansen, 2008) of a hidden Markov model is represented by the following two equations:

the state equation,

$$
X_{t},\left(X_{t-1}=x_{t-1}\right) \sim f\left(x_{t}, x_{t-1}\right)
$$

and the observation equation,

$$
Y_{t} \mid\left(X_{t}=x_{t}\right) \sim g\left(y_{t}, x_{t}\right) .
$$

Ahani Bridget is a Lecturer in the Department of Mathematics. Email her at: briggetk2002ng@yahoo.com. O. Abass is a Professor in the Department of Computer Science. Email him at: olabass@unilag.edu.ng.
The state variables $x_{t}$ and observations $y_{t}$ may be continuous-valued, discrete-valued or a combination of the two, $f\left(x_{t}, x_{t-1}\right)$, which indicates the probability density associated with moving from $x_{t-1}$ to $x_{t}$, and $g\left(y_{t}, x_{t}\right)$ are the state (transition) and observation densities. Practically, the $x$ 's are the unseen true signals in signal processing (Liu \& Chen 1995), the actual words in speech recognition (Rabiner 1989), the target features in a multitarget tracking problem (Avitzour 1995; Gordon, et al 1993; Gordon, et al 1995), the image characteristics in computer vision (Isard\& Blake 1996), the gene indicator in a DNA sequence analysis (Churchill 1989), or the underlying volatility in an economical time series (Pitt \&Shephard 1997). Hidden Markov Models represent the applications of dynamic state space model in DNA and protein sequence analysis (Krogh, et al 1994; Liu, et al 1997).

Using the functions provided by $\mathrm{C}++$ to expand an on-line algorithm for predicting a hidden Markov model, this articleutilizes Johansen (2009) SMCTC: Sequential Monte Carlo in $\mathrm{C}++$. Further supports were derived from results on predicted and actual data of 


\section{SEQUENTIAL MONTE CARLO APPROACH USING HIDDEN MARKOV MODELS}

monthly national air passengers in America (Zhang, et al., 2007). Cheng, et al. (2003) applied SMC methodology to the problems of optimal filtering and smoothing in hidden Markov models and SMC have also stirred great interest in the engineering and statistical literature (see Doucet, et al., 2000, for a summary). SMC methods have been applied for resolving a marginal Maximum Likelihood problem (Johansen, 2008) and Gordon, et al. (1993) applied SMC to optimal filtering. Herein the SMC method is developed for prediction of state by estimating the probability $\mathrm{p}\left(\mathrm{x}_{\mathrm{t} \mid} \mid \mathrm{y}_{1-\mathrm{t}-1}\right)$.

\section{Hidden Markov Models (HMM)}

Initially introduced and studied as far back as 1957 and into the early 1970's, HMM statistical methods have enjoyed more recent popularity.An HMM is a bivariate discrete-time process $\left\{X_{k}, Y_{k}\right\}_{k \geq 0}$ where $\left\{X_{k}\right\}_{k \geq 0}$ is a homogeneous Markov chain that is not directly observed, it can only be observed through $\left\{Y_{k}\right\}_{k \geq 0}$ that produces the observation. $\left\{Y_{k}\right\}_{k \geq 0}$, which is a sequence of independent random variables such that the conditional distribution of $Y_{k}$ only depends on $\mathrm{X}_{\mathrm{k}}$. The underlying Markov chain $\left\{X_{k}\right\}_{k \geq 0}$ is called the state. In general, the random variables $X_{k}$ and $Y_{k}$ can be of any dimension and of any domain, such as discrete, real or complex. $K$ elements of $X_{k}$ and $Y_{k}$ for $k=1,2, \cdots, K$ are collected to construct the vectors $X_{k}$ and $Y_{k}$, respectively. Due to the Markov assumption, the probability of the current true state given the immediately previous one is conditionally independent of the other earlier states:

$$
\mathrm{p}\left(\mathrm{x}_{\mathrm{k} \mid} \mid \mathrm{x}_{\mathrm{k}-1}, \mathrm{x}_{\mathrm{k}-2}, \cdots, \mathrm{x}_{0}\right)=\mathrm{p}\left(\mathrm{x}_{\mathrm{k} \mid} \mid \mathrm{x}_{\mathrm{k}-1}\right) .
$$

Similarly, the measurement at the $k^{\text {th }}$ time step is dependent only upon the current state, thus it is conditionally independent of all other states given the current state:

$$
\mathrm{p}\left(\mathrm{y}_{\mathrm{k} \mid} \mathrm{x}_{\mathrm{k}}, \mathrm{x}_{\mathrm{k}-1}, \cdots, \mathrm{x}_{0}\right)=\mathrm{p}\left(\mathrm{y}_{\mathrm{k} \mid} \mid \mathrm{x}_{\mathrm{k}}\right) .
$$

Using these assumptions the probability distribution over all states of the HMM can be written simply as:

$$
\begin{aligned}
& \mathrm{p}\left(\mathrm{x}_{0}, \cdots, \mathrm{x}_{\mathrm{k}}, \mathrm{y}_{1}, \cdots, \mathrm{y}_{\mathrm{k}}\right)= \\
& \quad \mathrm{p}\left(\mathrm{x}_{1}\right) \mathrm{p}\left(\mathrm{y}_{1 \mid} \mid \mathrm{x}_{1}\right) \prod_{\mathrm{k}=2}^{\mathrm{K}} \mathrm{p}\left(\mathrm{x}_{\mathrm{k} \mid} \mid \mathrm{x}_{\mathrm{k}-1}\right) \mathrm{p}\left(\mathrm{y}_{\mathrm{k} \mid} \mid \mathrm{x}_{\mathrm{k}}\right)
\end{aligned}
$$

which is reflected graphically as:

$$
y_{k} \sim g\left(y_{k}: x_{k}\right)
$$

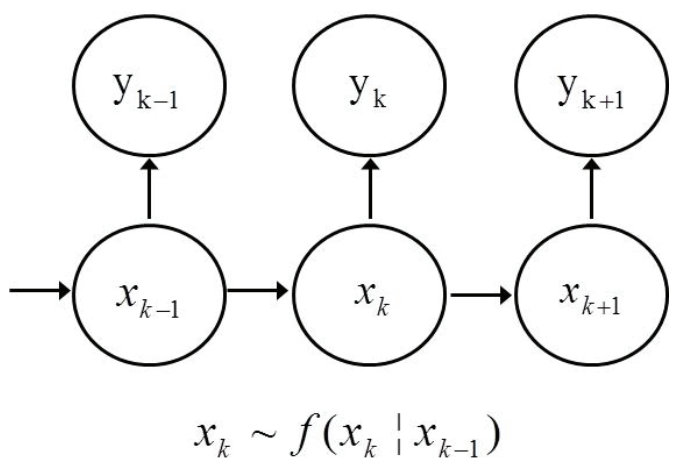

Given $p\left(x_{k-1}, y_{k-1}\right), p\left(x_{k}, y_{k}\right)$ can be found using the following prediction and update steps:

Prediction

$$
p\left(X_{k} \mid Y_{1: k-1}\right)=\int p\left(X_{k} \mid X_{k-1}\right) p\left(X_{k-1} \mid Y_{1: k-1}\right) d x_{-1}
$$

Update

$$
p\left(X_{k}: Y_{1: k}\right)=\frac{p\left(Y_{k} \mid X_{k}\right) p\left(X_{k} \mid Y_{1: k-1}\right)}{\int p\left(Y_{k}: X_{k}\right) p\left(X_{k} \mid Y_{1: k-1}\right) d x_{k}}
$$

In this case numerical integration is used, which becomes computationally complex when the number of states of $x_{k}$ are large: one particular Monte Carlo based approach to solve this for the HMM is the Sequential Monte Carlo Method (SMC).

Sequential Monte Carlo Methods (SMC)

Since their pioneering contribution in 1993 (Gordon, et al., 1993), SMC have become a well-known class of numerical methods for the 


\section{BRIDGET\& ABASS}

solution of optimal estimation problems in nonlinear non-Gaussian scenarios. The main idea of the SMC method is to represent the posterior density function $p\left(x_{0: k-1}, y_{0: k-1}\right)$ at time $k-1$ by samples and associated weights, $\left\{x_{0: k-1}^{(i)}, w_{0: k-1}^{(i)} i=1, \cdots, N\right\}$ and to compute estimates based on these samples and weights.As the number of samples becomes very large, this Monte Carlo characterization develops into an equivalent representation to the functional description of the posterior probability density function (Sanjeev, et al., 2002).

$$
\text { If }\left\{x_{0: k-1}^{(i)}, w_{0: k-1}^{(i)} i=1, \cdots, N\right\} \text { are samples }
$$

and associated weights approximating the density function, then $p\left(x_{0: k-1} \mid y_{0: k-1}\right)$, $\left\{x_{0: k-1}^{(i)}\right\}_{i=1}^{N}$ is a set of particles with associated weights $\left\{w_{0: k-1}^{(i)}\right\}_{i=1}^{N}$ with $\sum_{i=1: N} w^{(i)}=1$, and the density function are approximated by:

$$
p\left(\begin{array}{l|l}
x_{0: k-1} & \left.y_{0: k-1}\right) \approx \sum_{i=1}^{N} w_{k-1}^{(i)} \delta\left(x_{k-1}-x_{k-1}^{(i)}\right)
\end{array}\right.
$$

where $\delta(x)$ signifies the Dirac delta role, $y_{k}$ becomes available when a new observation arrives, and the density function $p\left(x_{k}, y_{k}\right)$ is obtained recursively in two stages:

1. Drawing samples $x_{k}^{i} \sim p\left(x_{k} x_{k-1}\right)$,

and

2. Updating the weight with the principle of importance sampling. (For details on SMC, see Doucel, et al., 2000; Sanjeev, 2002).

The particles are proliferated over time by Monte Carlo simulation to obtain new particles and weights (usually as new information are received), hence forming a series of PDF approximations over time. The reason that it works can be understood from the theory of (recursive) importance sampling.

\section{Procedural Functions \\ Methodology \\ Consider a particular algorithm for the} SMC, known also as the Sampling Importance Resampling (SIR) (Gordon, 1993; Carpenter, et al., 1999; Johansen, 2009). The algorithm can be summarized as follows: The algorithm is initiated by setting $k=1$, for which $p\left(x_{k}, x_{k-1}\right)=p\left(x_{k}\right)$ is defined.

Prediction for Step k:

Draw $N$ samples from the distribution $p\left(x_{k}, x_{k-1}=s_{k-1}^{(i)}\right) \quad \forall_{i}$ to form the particles $\left\{\hat{s}_{k}^{(i)}, \widetilde{w}_{k}^{(i)}\right\}_{i=1: N}$. The weight is $\widetilde{w}_{k}^{(i)}=\frac{\hat{w}_{k}^{(i)}}{\sum_{i} \hat{w}_{k}^{(i)}}$ where $\hat{w}_{k}^{(i)}$ is calculated from the conditional PDF $p\left(y_{k}: x_{k}=\hat{s}_{k}^{(i)}\right)$, given observation $Y_{k}$.

Resample for Step k:

Resample the random measure $\left\{\hat{s}_{k}^{(i)}, \widetilde{w}_{k}^{(i)}\right\}_{i=1: N}$ obtained in the prediction procedure to obtain $\left\{s_{k}^{(i)}, \frac{1}{N}\right\}_{i=1: N}$ which has uniform weights.

The importance of the prediction step is clear by establishing the following results.Using a importance function $q\left(x_{k}, y_{k}\right)$ satisfying the property

$$
q\left(x_{k}, x_{k-1}, y_{k}\right)=q\left(x_{k}, x_{k-1}, Y_{i}\right),
$$

$\left\{\hat{s}_{k}^{(i)}, \widetilde{w}_{k}^{(i)}\right\}_{i=1: N}$ is the random measure for estimating $p\left(x_{k}: y_{k}\right)$, where $\hat{s}_{i}=\left[\hat{s}_{1}^{(i)}, \cdots, \hat{s}_{k}\right\rfloor$ is the trajectoryfor particle $i$ and where $\widetilde{w}_{k}^{(i)}=\hat{w}_{k}\left(\hat{s}_{k}^{(i)}\right)$ is the normalized weights of particle $i$ at time $k$ which can be calculated recursively.

Let $\hat{w}_{k}^{(i)}=\hat{w}_{k}\left(\hat{s}_{k}^{(i)}\right)$, according to the argument at the $k^{\text {th }}$ step, the density function estimate for $p\left(x_{k}, y_{k}\right)$ is 


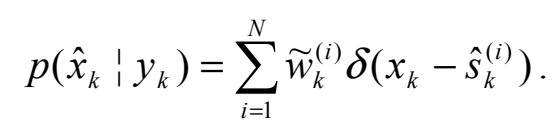

After the density function $\hat{p}\left(x_{k}, y_{k}\right)$ has been estimated, the observation prediction $\hat{y}_{k}$ with some samples with associated weights can be made. Accordingly, $\quad p\left(\hat{y}_{k}, y_{k-1}\right)$ are approximated by a new set of samples $\left\{\hat{y}_{k}^{1}, w_{k-1}^{(i)}\right\}_{i=1: N}$ and the observation prediction equation is:

$$
\hat{p}\left(\hat{y}_{k} \mid y_{k}\right)=\sum_{i=1}^{N} \widetilde{w}_{k}^{(i)} \delta\left(y_{k}-y_{k}^{(i)}\right) .
$$

\section{Data Description}

The above method is applied to the data sets of daily stock prices in the banking sector of the Nigerian Stock Exchange for price indices between the years 1 January 2005 to 31 December 2008 (see www.cashcraft.com/pricemovement.asp and Figure 1). Three hidden states are studied: bull, bear and even. These hiddenstates along with the observable sequences of large rise, small rise, no change, large drop and small drop were used to develop the hidden Markov model.The sequence of observation is obtained by subtracting the prior price from the current price, the percentage change then gives the classification of the sequence of observation, where $P_{t}$ is the price of an asset at time $t$, and the daily price relative/log returnis calculated as $r_{t}=\log p_{t} / p_{t-1}$.

Stock prices regularly alter in stock markets as observed in the price index on Tuesday, 5 February 2006; it fell by more than $100 \%$ (see Figure 2). No infallible system exists that indicates the precise movement of stock price. Instead, stock price is subjective to the influence of various factors, such as company fundamentals, external factors, and market behavior. These decide the state of the market which may be in bull or bear state. It grows along time through different market states, which are hidden states. The state of the market can be a Markovian process and is modeled in HMM.

Figure 1: Daily Stock Prices in the Banking Sector of the Nigerian Stock Exchange

(Price Index between the Years 1 January 2005 to 31 December 2008)

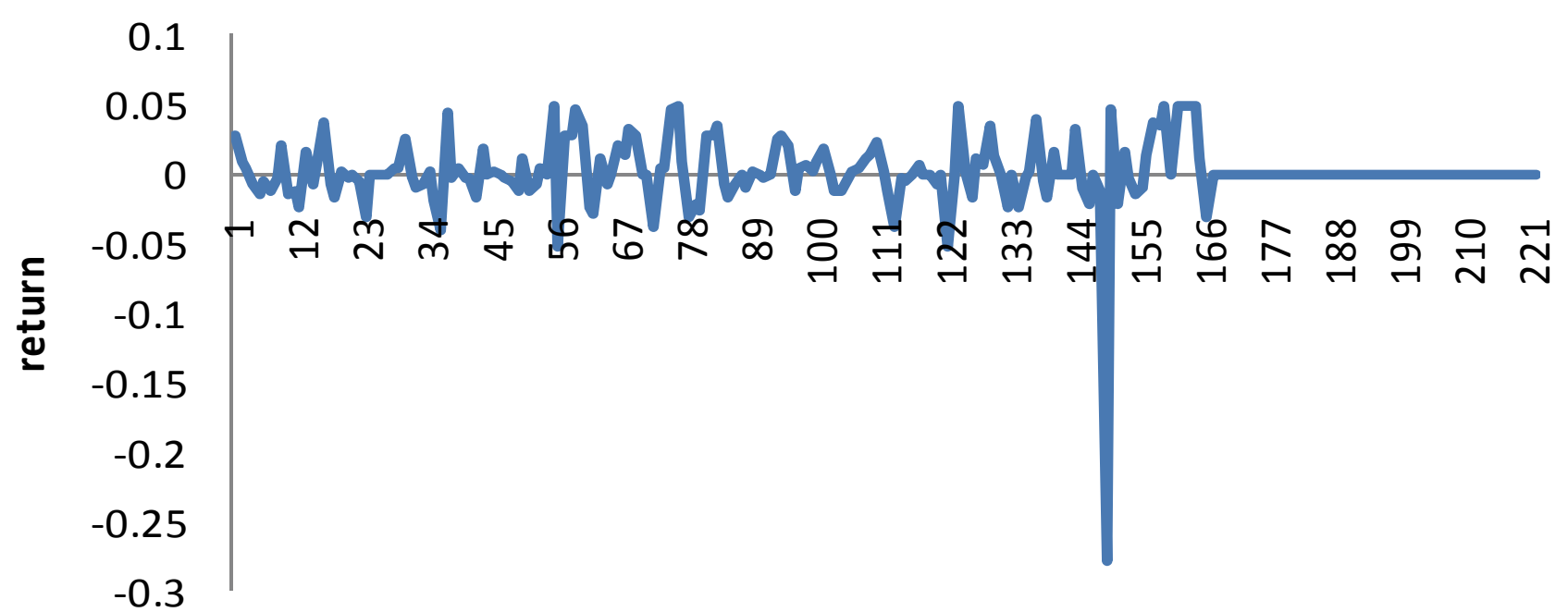

Time 


\section{BRIDGET\& ABASS}

Results

Using the functions provided by $\mathrm{C}++$, this study develops an on-line algorithm of predicting hidden Markov model (Johansen, 2009). The online prediction using SMC begins with states producing signals that follow the normal distribution. The numbers of hidden states in the Markov chain are defined as bull (state 1), even (state 2) and bear (state 3). Figure2 shows the predicted and actual daily stock prices and Table 1 shows predicted representational prices of the NSE and predicted errors.

The stock price is modeled in HMM and prediction is made based on available observations.Due to the strong statistical foundation of the HMM and SMC methods, the model can predict similar patterns proficiently (see Figure 2). Table 1 shows that the mean absolute percentage error (MAPE) is 0.068, hence, the predictive exactness is high.

\section{Conclusion}

An online sequential Monte Carlo method is used to predict ahidden Markov model. A C++ (Sequential Monte Carlo in $\mathrm{C}++$ ) template class library (Johansen, 2009) enabled the development of an online, sequential Monte Carlo for prediction. HMM and SMC method were introduced and the density function with a set of random samples with associated weights was approximated.Lastly, the data sets of daily stock prices in the banking sector of the Nigerian Stock Exchange were analyzed and experimental results revealed that the online algorithm is effective.

\section{References}

Avitzour, D. (1995). A Stochastic Simulation Bayesian Approach to Multitarget Tracking. Proceedings of the IEEE on Radar, Sonar and Navigation, 142, 41-44.

Carpenter, J. Clifford, P., \& Fearnhead, P. (1999). Improved Particle Filter for Nonlinear problems. Proceedings of the IEEE on Radar, Sonar and Navigation, 146, (1), 2-7.

Doucet, A., \& Johansen, A. M. (2008). A Tutorial on Particle Filtering and Smoothing. In Oxford Handbook of Nonlinear Filtering, D. Crisan \& B. Rozovsky, Eds. Oxford University Press.

Figure 2: Daily Stock Prices in the Banking Sector of the Nigerian Stock Exchange (Red line represents predicted stock price, Blue line represents actual stock price)

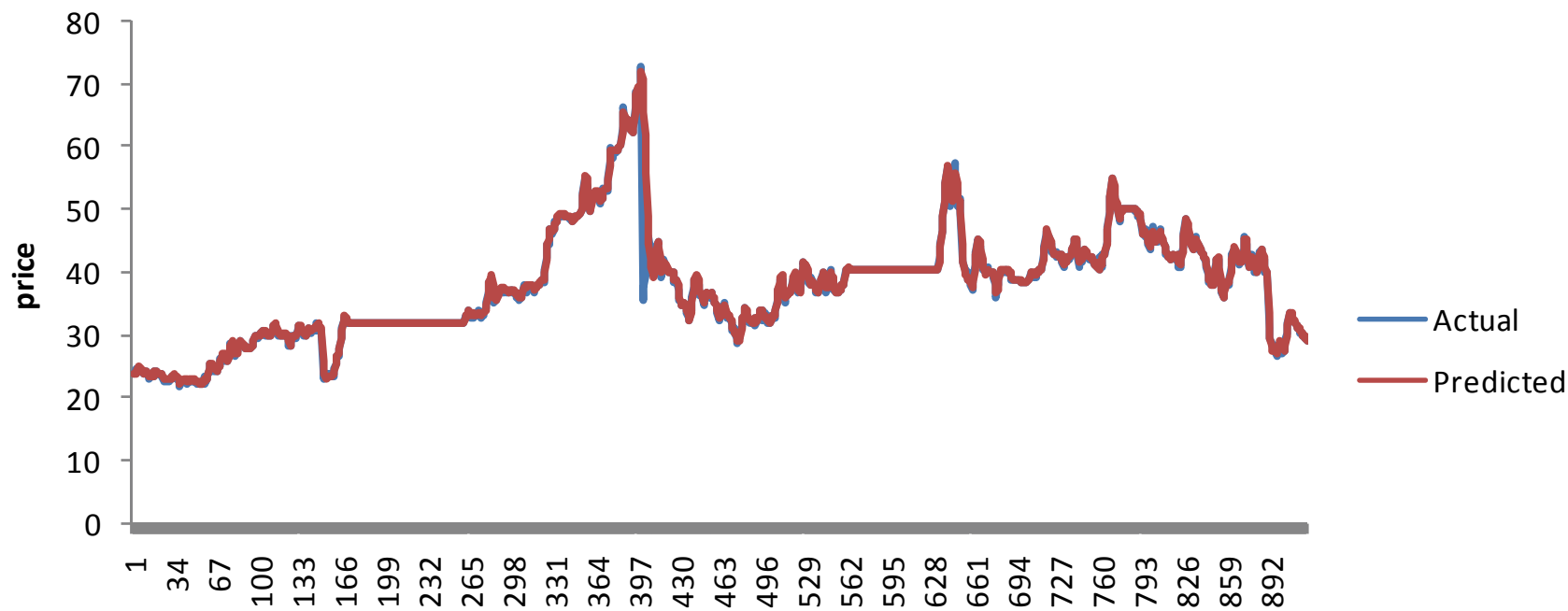

Time 
SEQUENTIAL MONTE CARLO APPROACH USING HIDDEN MARKOV MODELS

Table 1: Predicted Daily Stock Price in the Banking Sector of the NSE

\begin{tabular}{|c|c|c|c|c|c|c|}
\hline Actual & Predicted & R.E(\%) & MAPE(\%) & Actual & Predicted & R.E(\%) \\
\hline 24 & 23.8489 & 0.629583 & 0.068285 & 22.75 & 22.6411 & 0.478681 \\
\hline 24.7 & 24.0614 & 2.585425 & & 22.5 & 22.5232 & -0.10311 \\
\hline 24.9 & 24.4768 & 1.699598 & & 22.35 & 22.373 & -0.10291 \\
\hline 25 & 24.941 & 0.236 & & 22.45 & 22.3671 & 0.369265 \\
\hline 24.8 & 24.9793 & -0.72298 & & 22.46 & 22.4187 & 0.183882 \\
\hline 24.45 & 24.688 & -0.97342 & & 23.58 & 23.1687 & 1.744275 \\
\hline 24.3 & 24.3934 & -0.38436 & & 22.41 & 22.7752 & -1.62963 \\
\hline 23.99 & 24.0885 & -0.41059 & & 23.06 & 22.9608 & 0.430182 \\
\hline 23.95 & 23.933 & 0.070981 & & 23.7 & 23.5019 & 0.835865 \\
\hline 24.47 & 24.2088 & 1.06743 & & 24.8 & 24.4987 & 1.214919 \\
\hline 24.09 & 24.1513 & -0.25446 & & 25.68 & 25.5147 & 0.643692 \\
\hline 23.8 & 23.922 & -0.51261 & & 25.08 & 25.5347 & -1.813 \\
\hline 23.22 & 23.4166 & -0.84668 & & 24.4 & 24.9159 & -2.11434 \\
\hline 23.6 & 23.4176 & 0.772881 & & 24.7 & 24.7253 & -0.10243 \\
\hline 23.42 & 23.377 & 0.183604 & & 24.49 & 24.4938 & -0.01552 \\
\hline 23.6 & 23.4982 & 0.431356 & & 24.5 & 24.4089 & 0.371837 \\
\hline 24.49 & 24.1671 & 1.318497 & & 25.03 & 24.763 & 1.06672 \\
\hline 24.3 & 24.3828 & -0.34074 & & 25.4 & 25.2465 & 0.604331 \\
\hline 23.88 & 24.1404 & -1.09045 & & 26.24 & 26.0237 & 0.824314 \\
\hline 23.94 & 24.018 & -0.32581 & & 27 & 26.8721 & 0.473704 \\
\hline 23.85 & 23.89 & -0.16771 & & 27 & 27.2044 & -0.75704 \\
\hline 23.86 & 23.8301 & 0.125314 & & 26.98 & 27.2338 & -0.9407 \\
\hline 23.73 & 23.7339 & -0.01643 & & 26 & 26.5007 & -1.92577 \\
\hline 23 & 23.1971 & -0.85696 & & 26.09 & 26.1648 & -0.2867 \\
\hline 22.98 & 22.9523 & 0.12054 & & 26.17 & 26.0937 & 0.291555 \\
\hline 22.99 & 22.8886 & 0.441061 & & 27.39 & 26.8896 & 1.826944 \\
\hline 23 & 22.9326 & 0.293043 & & 28.75 & 28.2272 & 1.818435 \\
\hline 23 & 22.955 & 0.195652 & & 28.98 & 29.0147 & -0.11974 \\
\hline 23.1 & 23.055 & 0.194805 & & 28.07 & 28.6229 & -1.96972 \\
\hline 23.2 & 23.1768 & 0.1 & & 27.5 & 27.8895 & -1.41636 \\
\hline 23.78 & 23.6018 & 0.749369 & & 26.77 & 27.0194 & -0.93164 \\
\hline 23.7 & 23.7578 & -0.24388 & & 27.5 & 27.1466 & 1.285091 \\
\hline 23.45 & 23.6338 & -0.7838 & & 28.24 & 27.8034 & 1.546034 \\
\hline 23.3 & 23.4173 & -0.50343 & & 29.22 & 28.843 & 1.290212 \\
\hline 23.35 & 23.344 & 0.025696 & & 28.99 & 29.1623 & -0.59434 \\
\hline 22.89 & 23.0174 & -0.55657 & & 28.5 & 28.8644 & -1.2786 \\
\hline 22 & 22.2651 & -1.205 & & 28.31 & 28.5203 & -0.74285 \\
\hline 22.97 & 22.5771 & 1.710492 & & 28.3 & 28.3238 & -0.0841 \\
\hline 22.9 & 22.7748 & 0.546725 & & 28.02 & 28.0612 & -0.14704 \\
\hline 23 & 22.9519 & 0.20913 & & 28.08 & 27.9971 & 0.295228 \\
\hline 22.95 & 22.9895 & -0.17211 & & 28.05 & 27.9861 & 0.227807 \\
\hline 22.91 & 22.9678 & -0.25229 & & 27.95 & 27.9407 & 0.033274 \\
\hline 22.55 & 22.6986 & -0.65898 & & 27.91 & 27.9132 & -0.01147 \\
\hline 22.95 & 22.826 & 0.540305 & & 28.6 & 28.3646 & 0.823077 \\
\hline 22.94 & 22.8994 & 0.176983 & & 29.4 & 29.1204 & 0.95102 \\
\hline 23 & 22.9894 & 0.046087 & & 29.99 & 29.8659 & 0.413805 \\
\hline 22.98 & 23.0266 & -0.20279 & & 29.65 & 29.9393 & -0.97572 \\
\hline 22.94 & 23.0066 & -0.29032 & & 29.75 & 29.9012 & -0.50824 \\
\hline 22.8 & 22.8641 & -0.28114 & & 29.96 & 29.9926 & -0.10881 \\
\hline 22.51 & 22.6008 & -0.40338 & & 29.99 & 30.0266 & -0.12204 \\
\hline
\end{tabular}




\section{BRIDGET\& ABASS}

Churchill, G. A. (1989). Stochastic Models for Heterogeneous DNA Sequences. Bulletin of Mathematical Biology, 51, 79-94.

Gordon, N. J., Salmond, D. J., \& Smith, A. (1993). Novel Approach to Nonlinear/NonGaussian Bayesian State Estimation. IEEE Proceedings on Radar Signal Process, 140(2), 107-113.

Gordon, N. J., Salmon, D. J., \& Ewing, C. M. (1995). Bayesian State Estimation for Tracking and Guidance Using the Bootsrap Filter. Journal of Guidance, Control and Dynamics, 18, 1434-1443.

Isard, M., \& Blake, A. (1996). Contour Tracking by Stochastic Propagation of Conditional Density. In Computer Vision, Buxton \& R. Cipolla, Eds. New York: Springer. Doucet, A., de Freitas, J. F. G., \& Gordon, N. J. (2000). Sequential Monte Carlo Methods in Practice. New York: SpringerVerlag.

Johansen, A. M., Doucet, A., \& Davy, M. (2008). Particle methods for Maximum Likelihood Parameter Estimation in Latent Variable Models. Statistics and Computing, 18(1):47-57.
Johansen, A. M. (2009). Sequential Monte Carlo in $\mathrm{C}++$. Journal of Statistical Software, 30(6), http://www.jstatsoft.org/.

Krogh, A., Brown, M., Mian, S., Sjolander, K., \& Haussler, D. (1994).Proetein Modeling Using Hidden Markov Models. Journal of Molecular Biology, 235, 1501-1531.

Liu, J. S., \& Chen, R. (1995). Blind Deconvolution via Sequential Imputations. Journal of the American Statistical Association, 90, 567-576

Liu, J. S., Neuwald, A. F., \& Lawrence, C. E. (1997). Markov Structures in Biological Sequence Alignment. Technical Report, Stanford University.

Pitt, M. K., \&Shephard, N. (1997). Filtering via simulation: Auxiliar particle filters. www.nuff.ox.ac.uk/users/shephard.

Rabiner, L. R. (1989). A Tutorial on Hidden Markov Models and Selected Applications in Speech Recognition. Proceedings of the IEEE, 77, 257-286.

Sanjeev, A., Maskell, S., Gordon, N., \& Clapp, T. (2002). A tutorial on particle filter for on-line non-linear/non-Guassian Bayesian tracking. IEEE Transactions on Signal Processing, 50, 174-188. 